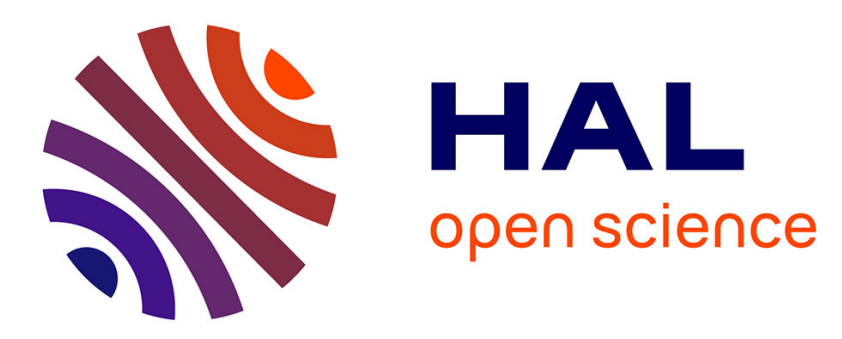

\title{
Progressive Lossless Mesh Compression Via Incremental Parametric Refinement
}

\author{
Sébastien Valette, Raphaëlle Chaine, Rémy Prost
}

\section{To cite this version:}

Sébastien Valette, Raphaëlle Chaine, Rémy Prost. Progressive Lossless Mesh Compression Via Incremental Parametric Refinement. Computer Graphics Forum, 2009, 8 (5), pp.1301-1310. 10.1111/j.1467-8659.2009.01507.x . hal-00533562

\section{HAL Id: hal-00533562 https://hal.science/hal-00533562}

Submitted on 9 Nov 2010

HAL is a multi-disciplinary open access archive for the deposit and dissemination of scientific research documents, whether they are published or not. The documents may come from teaching and research institutions in France or abroad, or from public or private research centers.
L'archive ouverte pluridisciplinaire HAL, est destinée au dépôt et à la diffusion de documents scientifiques de niveau recherche, publiés ou non, émanant des établissements d'enseignement et de recherche français ou étrangers, des laboratoires publics ou privés. 


\title{
Progressive Lossless Mesh Compression Via Incremental Parametric Refinement
}

\author{
Sébastien Valette ${ }^{1}$, Raphaëlle Chaine ${ }^{2}$ and Rémy Prost ${ }^{1}$ \\ Université de Lyon, CNRS \\ ${ }^{1}$ CREATIS-LRMN; UMR5220; Inserm U630; INSA-Lyon; Université Lyon 1, France \\ ${ }^{2}$ Université Lyon 1, LIRIS, UMR5205, F-69622, France
}

\begin{abstract}
In this paper, we propose a novel progressive lossless mesh compression algorithm based on Incremental Parametric Refinement, where the connectivity is uncontrolled in a first step, yielding visually pleasing meshes at each resolution level while saving connectivity information compared to previous approaches. The algorithm starts with a coarse version of the original mesh, which is further refined by means of a novel refinement scheme. The mesh refinement is driven by a geometric criterion, in spirit with surface reconstruction algorithms, aiming at generating uniform meshes. The vertices coordinates are also quantized and transmitted in a progressive way, following a geometric criterion, efficiently allocating the bit budget. With this assumption, the generated intermediate meshes tend to exhibit a uniform sampling. The potential discrepancy between the resulting connectivity and the original one is corrected at the end of the algorithm. We provide a proof-of-concept implementation, yielding very competitive results compared to previous works in terms of rateldistortion trade-off.
\end{abstract}

\section{Introduction}

Technological advances have pushed 3D graphics to higher levels of realism, and improvements are still emerging at a steady pace. The range of applications using 3D meshes for shape representation stretches from the visualization of topographic data on supercomputers to video games on cellular phones. With the eventual bandwidth limitations on such applications, 3D mesh compression has gained a lot of interest in the last 20 years. In this paper, we propose a novel 3D progressive lossless mesh compression paradigm, from which we derive an initial implementation (not suited for all the mesh classes) with the following characteristics:

- atomic granularity : starting from the coarse resolution mesh, new vertices are inserted in the triangulation one at a time, until the original mesh is completely reconstructed.

- the intermediate resolution meshes have their vertices well distributed over the surface.

- the mesh triangles exhibit good aspect ratio, as the mesh conforms to a local Delaunay criterion.

\section{Background on mesh compression}

The triangular mesh representation can be split in two different informations : geometry i.e. a 3D set of point coordinates, and connectivity i.e. a list of triangles connecting the points. Following Tutte's work [Tut62], the amount of information needed to encode the connectivity of a triangular mesh is bounded by the Tutte entropy $E_{\text {Tutte }}=3.245 \mathrm{bpv}$. The first works on mesh compression were done by Deering [Dee95], followed by the algorithm of Touma and Gotsman [TG98], where a canonical walk on the triangle mesh allows to encode the mesh connectivity by storing the vertices valences and non-frequent incidents codes. It has been shown to be very close to optimality [Got03]. Rossignac [Ros99] proposed an approach with a worst-case bound on its coding cost : Edgebreaker, which codes the mesh connectivity on 2 bits per triangle. Finally, Poulalhon and Schaeffer [PS06] have provided an approach based on Schneider tree decomposition, with a coding cost equal to the Tutte entropy. Single-resolution approaches usually combine one of the previously cited approaches or their derivatives for the mesh connectivity, and use the connectivity to improve geometry coding by means of prediction, the most widely 


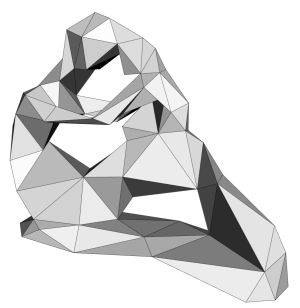

(a) base mesh $(100 v)$

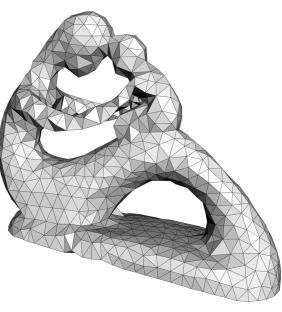

(b) $0.1 \mathrm{bpv}(1090 \mathrm{v})$

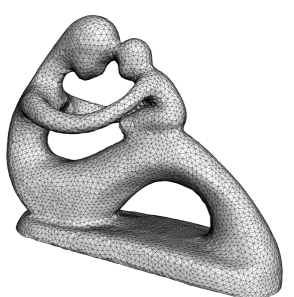

(c) $0.5 \mathrm{bpv}(8565 \mathrm{v})$

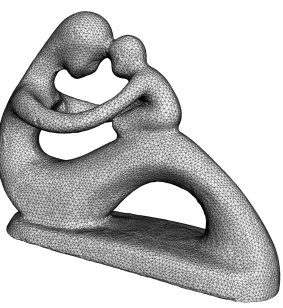

(d) $1 \mathrm{bpv}(17646 \mathrm{v})$

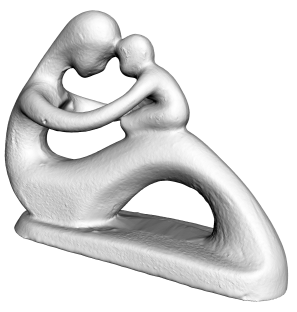

(e) $13.9 \mathrm{bpv}(241607 \mathrm{v})$

Figure 1: IPR-based Progressive transmission of the Fertility model (241k vertices). Note the uniform sampling and visual quality of the intermediate meshes $(b),(c)$ and $(d)$.

known being the parallelogram prediction. The vertices coordinates are usually quantized to 10 or 12 bits per coordinate, can be losslessly encoded, such as proposed by Isenburg et al. [ILS05], and can be used to predict the connectivity, thus improving compression rates $\left[\mathrm{LCL}^{*} 06\right]$.

Progressive compression approaches start with the coding of a coarse approximation of the original mesh and add information allowing to progressively reconstruct the original mesh by means of refinements. First approaches have focused on connectivity refinement : Cohen-or et al. [COLR99] start from the original mesh and iteratively remove its vertices in carefully selected batches until the lowest resolution is obtained. The reconstruction is guaranteed by a 4-colour coding scheme. Taubin et al. [TGHL98] proposed a tree-based refinement scheme called progressive forest split. The vertex-split based progressive meshes scheme proposed by Hoppe [Hop96] has been used in [PR00] and [KBG02] to encode vertex splits in batches. When one only wants to store the shape of the object represented by the mesh, the connectivity can be changed to a much simpler one, a structured connectivity, whose coding cost is negligible compared to an irregular connectivity and is a perfect fit for geometry compression via transform coding. Efficient progressive geometry compression using uniform subdivision was first proposed by Khodakovsky et al. [KSS00] and Guskov et al. [GVSS00]. Payan and Antonini [PA05] proposed an optimized bit-allocation algorithm within this framework. Gu et al. [GGH02] proposed to resample the original mesh to a regular connectivity mesh, a geometry image. This work was further improved by Peyre and Mallat [PM05] by taking into account local anisotropy in the shape to be encoded. An other approach using transform coding is the spectral compression introduced by Karni and Gotsman [KG00]. When the original mesh connectivity matters, using transform coding algorithms for geometry compression is not straightforward, as these methods are based on regular mesh subdivision. To solve the problem faced when compressing irregular connectivity, Alliez and Desbrun [AD01] try to reverse $\sqrt{3}$ subdivision on the input mesh [Kob00], while the Wavemesh coder proposed by Valette and Prost [VP04] tries to reverse face quadrisection. For both approaches, when the regular subdivision is not applicable, incident codes need to be transmitted and generate coding overhead. As a consequence, compression efficiency of these approaches is highly dependent on the regularity of the input mesh. Note that these approaches try to keep the mesh connectivity as regular as possible during the simplification step, but finding the optimal simplification rules is still an open problem. Finally, the most recent progressive coders are not only driven by connectivity refinement, but also by geometric measures on the reconstructed mesh. Gandoin and Devillers [GD02] propose to encode the vertices coordinates with a Kd-tree coder, with the side-effect of efficient connectivity compression by means of generalized vertex splits. This approach allows complex connectivity operations and is therefore able to handle meshes with complex topology such as polygon soups. Peng and Kuo [PK05] further improved this approach by replacing the Kd-tree with an octree data structure. The octree cells are refined in a prioritized order, where the cells subdivisions offering the best distortion improvement are performed first. These approaches provide good results for lossless compression, but they are based on structured volume hierarchies which induce anisotropy and blocking artifacts, thus reducing rate-distortion performance at low bitrates (figure 2.(c)).

\section{Outline of our approach}

We propose to redefine the problem of progressive mesh transmission into a mesh generation problem. Figure 2 shows a comparison between our Incremental Parametric Refinement (IPR) approach, Wavemesh [VP04] and the Octree Compression method [PK05]. At 1bpv, our approach generates a visually pleasing mesh, while the reconstruction with Wavemesh at $1.42 \mathrm{bpv}$ exhibits stretched triangles and a higher approximation error $(0.6 \%$ of the model Bounding Box Diagonal Length). At 1bpv, the Octree Compression approach outputs a mesh with the worse visually pleasing properties and higher approximation error $(0.8 \%)$. The problem of surface meshing has been widely studied in the last years [AUGA07]. We use a refinement scheme driven by a Delaunay mesh generation approach, which produces uni- 


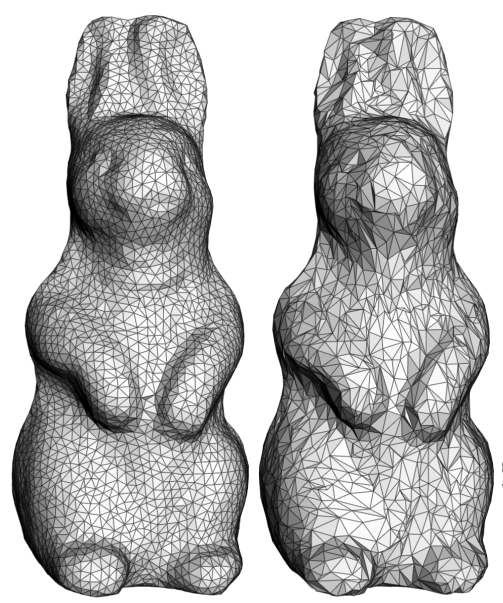

(a) IPR: $0.3 \%$

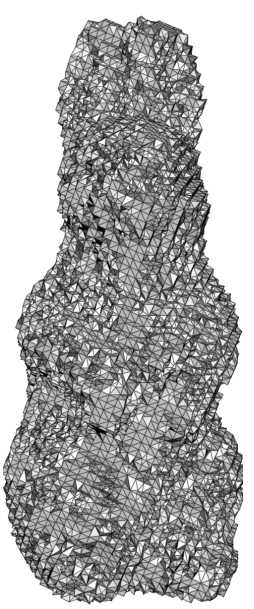

(c) $\mathrm{OC}: 0.8 \%$
Figure 2: the rabbit model compressed down to 1 bit per original vertex with our approach (a) and the Octree Compression (c), and at 1.42 bpv with Wavemesh (b). The percentages represent the approximation error in terms of Hausdorff distance, with regard to the mesh bounding box diagonal length.

form triangulations during progressive transmission at low coding cost, as most of the connectivity is implicitly defined by the reconstruction algorithm. Note that an alternate approach using reconstruction algorithms has been proposed by Chaine et al. [CGR07] to predict the mesh topology, but it is limited to connectivity encoding, assuming that the vertices coordinates are already available. Our approach simultaneously encodes the vertices coordinates and the mesh connectivity and avoids the use of any volumic data structure. Subsequently, we note the input mesh as $\mathcal{M}$ and $n$ its number of vertices. We assume in this paper that the final reconstructed mesh will have the same connectivity as the original mesh, and each vertex coordinate will be quantized to a given number $N_{q}$ of bits. The coding will consist in starting from a base mesh $\mathcal{M}_{b}$ where $b$ is its number of vertices $(b<<n)$. The set of vertices of $\mathcal{M}_{b}$ is a subset of the vertices of $\mathcal{M}$, and their coordinates are quantized on $N_{b}$ bits $\left(N_{b}<N_{q}\right)$. Afterwards, $\mathcal{M}_{b}$ is iteratively refined by inserting the missing vertices in order to construct a sequence of meshes $\mathcal{M}_{b}, \mathcal{M}_{b+1}, \mathcal{M}_{b+2}, \ldots, \mathcal{M}_{n-2}, \mathcal{M}_{n-1}, \mathcal{M}_{n}$

Finally, when all the vertices of $\mathcal{M}$ have been transmitted, we need to ensure that the connectivity of the reconstructed mesh is the same as the original. This is done by iteratively flipping some edges of $\mathcal{M}_{n}$ until it matches $\mathcal{M}$.

Our scheme takes into account the following assumptions:

- A fine to coarse approach for connectivity encoding decreases coding efficiency, as only the final mesh connectivity has to conform to the input mesh connectivity. In- stead, a coarse to fine approach can improve compression at low bitrates, as when the current mesh $\mathcal{M}_{i}$ has relatively few vertices $(i \ll n)$, every operation should create a new vertex (and never require incident codes), similarly to uniform subdivision schemes.

- In spirit with the octree-based coder [PK05], the efficiency of progressive transmission can be improved with a careful prioritization of refinement sites.

- For each resolution level $\mathcal{M}_{i}$ where $b \leq i \leq n$, we need to provide a good trade-off between the level of quantization of the vertices coordinates and the number of vertices in the mesh, as pointed by King and Rossignac [KR99]. Hence, if the final vertices are coded on $N_{q}$ bits, we can encode the vertices of coarser resolution levels with a lower number of bits. The remaining bits with low significance are transmitted latter in the refinement sequence, similarly to the SPIHT coder [SP96].

For this aim, we propose a decoder-centric template Incremental Parametric Refinement (IPR) algorithm which is instantiated with output writing operations for the compression step, and input operations for the decompression. This algorithm performs a good balance between vertices quantization and connectivity evolution at each resolution level $\mathcal{M}_{i}$ with two independent measures:

- An estimate of the adequate number of bits needed to represent the coordinates of a given vertex. This number is computed by IPR and grows during refinement until full precision is reached.

- A prediction of the refinement sites. At each resolution level $i$, the algorithm can predict which region is a good candidate for refinement i.e. the location where increasing the sampling density is likely to lower the approximation error.

\section{Description of the IPR algorithm}

In this section, we develop the idea behind our paper : For each resolution level, IPR carefully chooses the location of the next refinement, and transmits the coordinates of the vertex used for the refinement, using differential coding. We exploit the statistical fact that a way to reduce the approximation error for a given sampling is to refine the regions having the lowest sampling density. Also, at any time, the algorithm can request some data to refine the coordinates of a given vertex. Again, compression and decompression are performed using the same algorithm, except that compression writes data and decompression reads the data. As a consequence, the data requests do not need explicit communication between the decoder and the encoder, only the requested data needs to be transmitted in the bitstream.

\subsection{A new surface-aware refinement scheme}

We propose an incremental refinement scheme which aims at constructing uniform triangulations by means of simple 


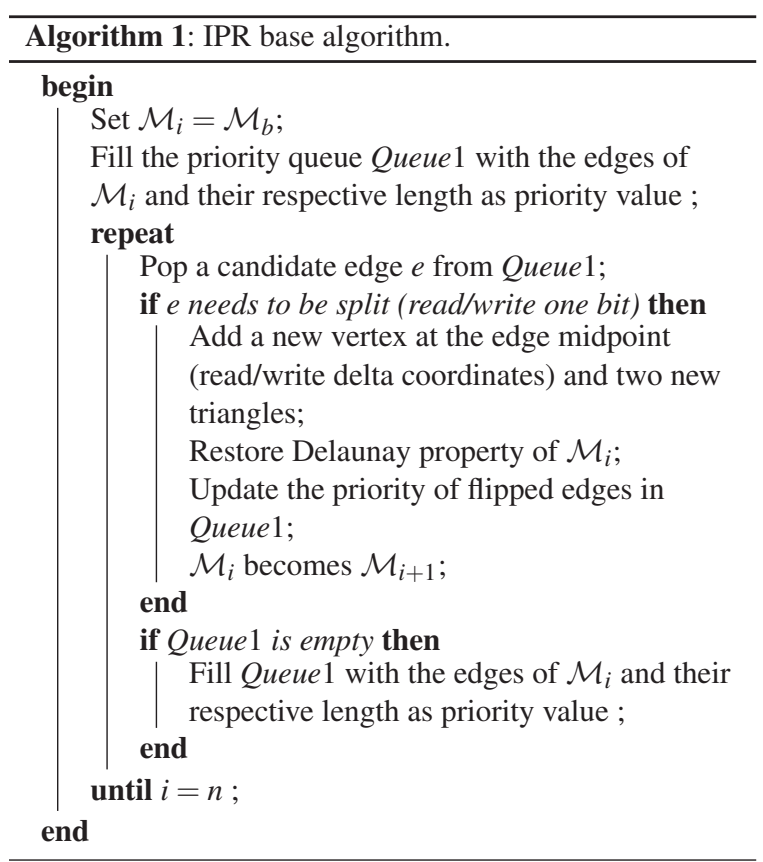

atomic operations. Each operation consists in an edge split followed by an automatic local Delaunay enforcement step, as shown by figure 3. At a given resolution level $i$, we pick the longest edge $e$ of $\mathcal{M}_{i}$, in order to increase the sampling density of the surface (figure 3.(b)) while preserving uniformity. This operation requires the insertion of a new vertex $v_{i}$ into the triangulation. The position of $e$ does not need to be transmitted, as it is computed by both the decoder and encoder. At the encoder end, $v_{i}$ is chosen among the vertices of $\mathcal{M}$ with the goal of getting the best approximation improvement.

Then, after each vertex insertion, the quality of the triangulation is increased, using a local Delaunay criterion, such as proposed by Dyer et al. [DZM07]. This consists in performing local checks on all modified edges and their neighboring edges: for each edge $e$ to check, we measure the Delaunay criterion $D_{e}=\alpha+\beta-\pi$, where $\alpha$ and $\beta$ are the values of the opposite angles of $e$. A positive value for $D_{e}$ means that $e$ is not a local Delaunay edge and therefore has to be flipped. Note that flipping an edge can also change its neighboring edges Delaunay criterion value. Consequently, we also have to check that the Delaunay criterion is also respected for this neighborhood and repeat these operations until all the edges satisfy the criterion. The whole scheme is easy to implement using a priority queue, and its convergence is guaranteed [DZM07]. An efficient way of keeping track of the longest edge in $\mathcal{M}_{i}$ is to use an other priority queue whose average construction and update complexity is in $n \log n$.

Moreover, we prevent the edge flips that would cause the

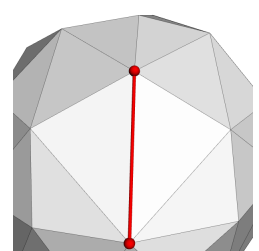

(a)

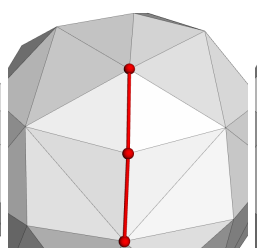

(b)

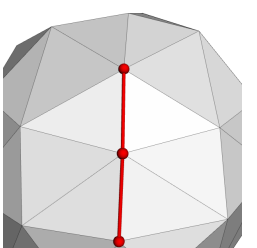

(c)
Figure 3: the proposed refinement scheme : (a) an edge e (in bold red) is selected for refinement. (b) It is split in two, resulting in the creation of a new vertex and two new triangles. (c) The resulting triangulation is modified in order to satisfy a local Delaunay Property, by means of edge flips. In this example, two edges have been flipped.

underlying geometry to change significantly. For each edge $e$ to be flipped, we measure the volume $v_{e}$ and area $a_{e}$ of the tetrahedron formed by the two vertices of $e$ and the two vertices opposite to $e$, and compute the flip criterion $F_{e}=$ $\frac{\sqrt[3]{v_{e}}}{\sqrt{a_{e}}}$. Whenever $F_{e}$ is above a given threshold $F_{t}$, we forbid the flip. In our experiments, we set $F_{t}=0.3$.

Finally, the cost of one refinement operation is reduced to the localization of the edge to split and the additional geometric information needed to create the new vertex. Connectivity evolves automatically, without any overhead data.

Our refinement algorithm takes into account the geometric properties of the evolving reconstructed mesh, but the operations and criteria used here are only performed in the mesh parametric domain, in contrast with volumetric subdivision approaches such as [GD02, PK05], hence the name Incremental Parametric Refinement. This scheme allows for faithful progressive approximation of the original model, even at low bitrates.

\subsection{Predicted splits for connectivity encoding}

The proposed scheme can encode the mesh connectivity in a very efficient way. The challenge here is to decide for a resolution level $i$ whether the longest edge $e$ needs to be split or not at the decoder end. This implies a mapping between the original mesh vertices and the reconstructed mesh edges. Each edge $e_{j}$ of $\mathcal{M}_{i}$ will be associated with a set $\mathcal{V}_{j}$ of vertices in $\mathcal{M}$ not present in $\mathcal{M}_{i}$ but which are candidates for insertion by splitting $e_{j}$ in $\mathcal{M}_{i}$. As the resolution level increases, the set $\mathcal{V}_{j}$ contains less and less vertices, and will eventually become empty. As a result, when the set is empty, we need to prevent the edge split. Note that at a given resolution level, it can happen that the set of candidates $\mathcal{V}_{j}$ for $e_{j}$ is empty, but it can later be given new candidate vertices after edge flips occur in its neighborhood.

Therefore, for each selected edge $e$, we need to transmit one bit ( 1 for yes, 0 for no), so that the decoder knows 


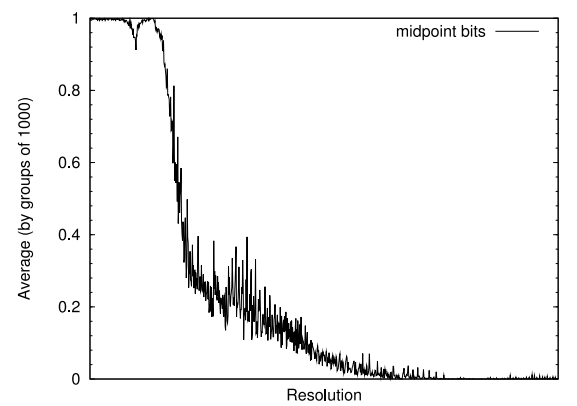

Figure 4: Values of midpoint codes when compressing the Fertility model: the moving average value is very close to 1 at the beginning of the transmission and gradually vanishes as the resolution increases.

whether $e$ must be split or not. Subsequently, we will call these informations midpoint codes. If the picked edge is not split, the same test is performed with the next longest edge in the queue, until a to-be-split edge is encountered. After each split, the resolution level is incremented. At low resolution levels, the midpoint codes will most frequently have the value 1 , and will most often be 0 at high resolution levels. This is illustrated by figure 4 which shows the midpoint codes average values, by order of appearance, computed for groups of 1000 successive values when compressing the Fertility model. We can then improve the compression efficiency using adaptive arithmetic coding with a relatively short statistics update interval (the so-called rescale interval). In our experiments, we have set the rescale interval to 15 . As an example, when compressing the fertility model, 987141 midpoint codes are needed. The entropy of this bitstream is 0.89 bits per midpoint code, but using adaptive coding reduces the coding cost to 0.36 bits per midpoint code. This translates to a relative coding cost of 1.45 bits per vertex.

\subsection{Quantization/connectivity trade off}

The quantization of vertices coordinates plays a key role for efficient mesh compression. Indeed, it is well known that the size of the geometric information is much bigger than the size of connectivity information. As explained in section 2, connectivity-driven compression approaches such as Wavemesh usually encode and transmit vertices coordinates in a constant-quantization way, whereas recent compression schemes such as the Octree Compression approach refine the vertices coordinates as the resolution increases. But Octree and Kd-tree approaches still do not provide the good balance between the number of vertices in the mesh and the level of quantization of vertices coordinates. This is clearly illustrated by figure 2.(c) : at $1 \mathrm{bpv}$, the Octree Compression delivers a mesh made of 12959 vertices, but the vertices coordinates quantization is too coarse to provide a good ap-

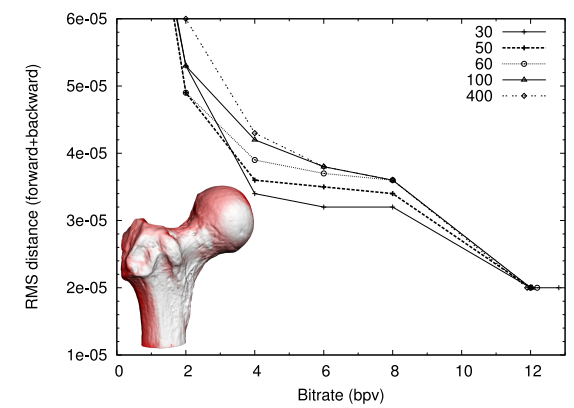

Figure 5: comparison of rate-distortion performance when compressing the balljoint model, for various values of $q_{t}$

proximation of the rabbit model. Instead, in figure 2.(a) our approach reconstructs a more accurate mesh with only 4833 vertices, but with a higher number of bits transmitted for the vertices coordinates. At this resolution level, $78 \%$ of the vertices coordinates are coded with 11 bits precision, and the remaining are coded with 12 bits. More generally, each vertex $v_{i}$ is given a specific quantization level $Q_{i}$ with $0<Q_{i}<Q_{m}$ where $Q_{m}$ is the maximal quantization level (usually 12 bits). The quantized coordinates $\widetilde{c_{i}}$ of a given vertex $v_{i}$ are computed as:

$$
\widetilde{c_{i}}=\left\lfloor 2^{Q_{i}-Q_{m}} c_{i}\right\rfloor
$$

We propose to first transmit the coordinates of the base mesh vertices with a low number of bits $\left(N_{b}=4\right.$ in our experiments), and let the IPR algorithm choose whether the quantization level $Q_{i}$ is sufficient or not for a given vertex $v_{i}$. This is done by measuring the squared distance $D_{i}$ (in its quantized version) between $v_{i}$ and its closest neighbor vertex $v_{j}$ :

$$
D_{i}=\left\|\widetilde{c_{i}}-\widetilde{c_{j}}\right\|^{2}
$$

Where $c_{i}$ are the coordinates of the vertex $v_{i}$. Whenever $D_{i}$ is lower than a threshold $q_{t}$, the quantization precision is increased by one bit (if full precision has not already been reached). This results in transmitting one bit of refinement for each coordinate of $v_{i}$. When a new vertex $v_{i}$ is inserted in $\mathcal{M}_{i}$ by splitting the edge $e$, its quantization level is set to the average between the quantization levels of the vertices of $e$, and only the difference between the edge midpoint and the actual coordinates of $v_{i}$ is transmitted, thus reducing the entropy of the data to transmit. The resulting delta-coordinates are entropy coded by means of arithmetic coding. Eventually, when a new vertex is inserted in the mesh, the quantization criterion will not be satisfied, and the coordinates of surrounding vertices will be refined. When all the mesh vertices are inserted i.e. when $\mathcal{M}_{n}$ is reconstructed, we refine the vertices coordinates which have not been transmitted with the desired final precision.

As a simple example, let us assume a 1D case were we as- 
sume that the coordinates to encode are between 0 and 127, with $q_{t}=600$ : two vertices $v_{1}$ and $v_{2}$ with coordinates 80 (1010000 in binary) and 30 (0011110 in binary) are currently coded with a precision of 4 bits, which results in quantized values of 10 (1010 in binary) and 3 (0011 in binary), the 4 most significant bits for both coordinates. The squared difference between this numbers is 49 , which is less than $q_{t}$. Increasing the precision to 6 bits will make the coordinates become 40 (101000 in binary) and 15 (001111 in binary) which satisfy the quantization criterion, as $(40-15)^{2}=625$.

Figure 5 shows the rate-distortion compression curves obtained with our approach when compressing the balljoint model, for various values of $q_{t}$. This parameter improves the efficiency of our approach by about $20 \%$ at intermediate level, but also increases the amount of data needed for the final lossless compression. In our experiments, we have set $q_{t}$ to the conservative value of 600 , which still offers a gradually increasing precision with no significant overhead for lossless compression.

Finally, on figure 1.(b), the reconstruction of the fertility model at a bitrate of $0.1 \mathrm{bpv}$ has 1090 vertices. $54 \%$ of the coordinates are encoded with 10 bits, $43 \%$ with 11 bits, and the remaining $2 \%$ with more precision. This results in an RMS error of 1.96 while constant quantization (when $N_{b}=Q_{m}=12$ ) results in an error of 3.45 for the same bitrate. For the rabbit model at $1 \mathrm{bpv}$, adaptive quantization results in an error of 4.4 while constant quantization leads to an error of 5. Moreover, figure 9 (right) shows that for bitrates below $2 \mathrm{bpv}$, adaptive quantization improves compression compared to constant quantization.

\subsection{Getting the original connectivity back}

When the highest-resolution mesh $\mathcal{M}_{n}$ is reconstructed, we still need to modify its connectivity $\mathcal{T}_{n}$ so that it matches the triangulation of $\mathcal{M}$, noted $\mathcal{T}$. The problem of finding a finite sequence of edge flips to change a genus- 0 triangulation into an other genus- 0 triangulation has been solved for the unlabeled case by Wagner [Wag36] and by Gao et al. [GUW01] for the labeled case (our case) but finding the flip sequence with minimal size is still an open problem. Currently available solutions use a canonical triangulation as a mandatory intermediate connectivity on the path between $\mathcal{T}_{n}$ and $\mathcal{T}$ and therefore perform a high number of flips. As we want the smallest number of flips as possible for compression efficiency reasons, instead of using an of-the-shelf algorithm, we propose to use an heuristic approach, taking into account the fact that in our case, $\mathcal{T}_{n}$ and $\mathcal{T}$ should be very similar. We define an asymmetric measure of similarity $S$ between two triangulations $\mathcal{T}_{1}$ and $\mathcal{T}_{2}$ as:

$$
S\left(\mathcal{T}_{1} \rightarrow \mathcal{T}_{2}\right)=\sum_{e_{i} \in \mathcal{E}_{2}} d_{\mathcal{T}_{1}}\left(e_{i, 1}, e_{i, 2}\right)
$$

where $\mathcal{E}_{2}$ is the set of edges of $\mathcal{T}_{2} . e_{i, 1}$ and $e_{i, 2}$ are the vertices of the edge $e_{i} . d_{\mathcal{T}_{1}}\left(e_{i, 1}, e_{i, 2}\right)$ is the flip distance between
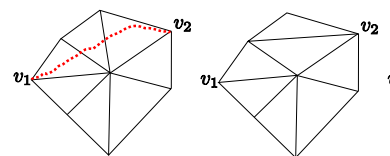

$\mathcal{T}_{1}$

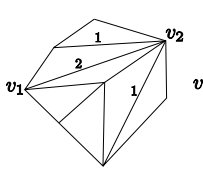

$\mathcal{T}_{2}(S=4)$

(a)

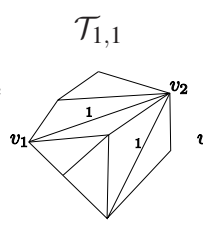

$\mathcal{T}_{2}(S=2)$

(b)

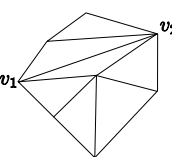

$\mathcal{T}_{1,2}$

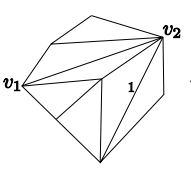

$\mathcal{T}_{2}(S=1)$

(c)

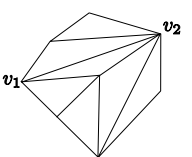

$\mathcal{T}_{1,3}$

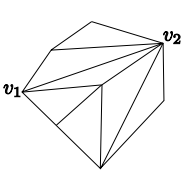

$\mathcal{T}_{2}(S=0)$

(d)
Figure 6: Measuring the flip distance between two vertices: the triangulations $\mathcal{T}_{1}$ (top) and $\mathcal{T}_{2}$ (bottom) have the same set of vertices, but different connectivity. We measure the flip distance for each edge of $\mathcal{T}_{2}$. As an example, when measuring the flip distance $d_{\mathcal{T}_{1}}\left(v_{1}, v_{2}\right)$ the shortest path between $v_{1}$ and $v_{2}$ crosses two edges ( $a$, top). There are only three edges in $\mathcal{T}_{2}$ with a non-null flip distance, and $S\left(\mathcal{T}_{1} \rightarrow \mathcal{T}_{2}\right)=4$. Afterwards, we perform three edge flips in $\mathcal{T}_{1}(b)(c)(d)$ to change $\mathcal{T}_{1}$ into $\mathcal{T}_{1,3}=\mathcal{T}_{2}\left(S\left(\mathcal{T}_{1} \rightarrow \mathcal{T}_{2}\right)=0\right)$

$e_{i, 1}$ and $e_{i, 2}$ in $\mathcal{T}_{1}$ i.e. the number of flips needed to change $\mathcal{T}_{1}$ into a triangulation where the edge $e_{i}$ exists. We compute this number with an algorithm similar to Dijkstra's shortest path algorithm, where the path does not follow the mesh edges, but crosses them by walking on the dual graph. The flip distance is then the number of crossed edges, which all have to be flipped when one wants to create the edge $e_{i}$, as shown in figure 6.(a). Note that there can exist several minimal paths between two vertices, but this is not an issue, as with our similarity measure, we only consider the lengths of the paths and not the edges they come across. Then, we perform edge flips on $\mathcal{T}_{n}$ with the objective to decrease $S\left(\mathcal{T}_{n} \rightarrow \mathcal{T}\right)$. This translates to reducing the flip distances of the edges in $\mathcal{T}$ by flipping the edges in $\mathcal{T}_{n}$.

For each edge of $\mathcal{M}_{n}$, the algorithm transmits a single bit informing whether the edge should be flipped or not. On the encoder end, the decision is taken based on the effect of the edge flip on $S\left(\mathcal{T}_{n} \rightarrow \mathcal{T}\right)$. If this value decreases, the edge $e_{i}$ is flipped (coded 1). If it increases, $e_{i}$ is not flipped (coded 0). If the value is the same for both cases, the encoder performs a supplementary test : it computes the value $\left.\left.d_{\mathcal{T}}\left(e_{i, 1}, e_{i, 2}\right)\right)-d_{\mathcal{T}}\left(e_{i, 1}^{\prime}, e_{i, 2}^{\prime}\right)\right)$ where $e_{i}^{\prime}$ is the edge created when flipping $e_{i}$ i.e. it checks whether flipping $e_{i}$ will decrease its flip distance (measured this time by walking on $\mathcal{T})$. If the flip distance is decreased, $e_{i}$ is flipped. Note that for practical reasons, we actually do not compute all the flip distances in the mesh when computing $S\left(\mathcal{T}_{n} \rightarrow \mathcal{T}\right)$, but only the flip distances of the edges incident to the four vertices involved in the edge flip.

After all the edges have been visited, the encoder checks whether the two meshes are identical, by simply verifying 
that $S\left(\mathcal{T}_{1} \rightarrow \mathcal{T}_{2}\right)=0$. If they are identical, the encoder puts a 1 in the bitstream, otherwise a 0 and the decoder will repeat the traversal on all the edges. This procedure is repeated until the two meshes are identical. In our experiments, the number of loops on the edges is usually between 2 and 5 . The number of loops directly influences the bitstream entropy of our algorithm. As an example, for the Fertility model, 3 loops were required to reconstruct the original connectivity, with a relative coding cost of $2.82 \mathrm{bpv}$ using arithmetic coding. In figure 6, (a), (b) (c) and (d) show the complete set of flips needed to change $\mathcal{T}_{1}$ into $\mathcal{T}_{2}$. the bottom part show the flip distances computed on $\mathcal{T}_{2}$ during this transformation, and give the value of $S=S\left(\mathcal{T}_{1} \rightarrow \mathcal{T}_{2}\right)$.

Unfortunately, there is no guaranteed convergence for this algorithm and no proof of convexity for $S$. We experienced non-convergence only when the chosen order for vertices insertion was clearly wrong, resulting in a reconstructed mesh with normal flips and possibly degenerated connectivity. Therefore, stress has to be put on keeping the meshes $\mathcal{M}_{i}$ as close as possible to the original mesh $\mathcal{M}$ during the selection of transmitted vertices and on avoiding connectivity drifts that can be induced with the current choice of the vertices.

\subsection{Picking the right vertices order of insertion}

For each resolution level $i$, the new vertex to insert in $\mathcal{M}_{i}$ is chosen by the encoder, so as to decrease the approximation error as much as possible. This requires a mapping between $\mathcal{M}$ and $\mathcal{M}_{i}$, such as MAPS [LSS*98] or the approach of Schreiner et al. [SAPH04]. However, the dynamic edge flips performed during the iterative reconstruction of $\mathcal{M}_{i}$ prevent the use of such approaches in a computationally efficient way. As a first approximation, we propose a fast approach to compute the list of candidate vertices when an edge $e_{i}$ is to be split. We first use a geodesic Voronoi diagram on $\mathcal{M}$, with the vertices of $\mathcal{M}_{i}$ as Voronoi sites. This provides a good mapping between the vertices of $\mathcal{M}$ and the vertices of $\mathcal{M}_{i}$ for each resolution level $i$ (figure 7.(b)).

Each Voronoi Region $V_{j}$ is then a set of vertices of $\mathcal{M}$ associated to a given vertex $v_{j}$ of $\mathcal{M}_{i}$. Afterwards, we associate each vertex $v_{k}$ inside a region $V_{j}$ to the edge $e$ incident to $v_{j}$ giving the maximal value for the following criterion :

$$
C(e)=\frac{\vec{e}}{\|\vec{e}\|} \bullet \overrightarrow{v_{j} v_{k}}
$$

i.e. we associate $v_{k}$ to its closest edge. The resulting mapping can be seen on figure 7.(c). When a vertex $v_{k}$ has been associated to an edge $e$, it can be associated to one of the two triangles incident to $e$ according to its position with regards to the plane bisecting the two triangles. The result of such mapping can be seen in figure 7.(d). Finally, the list of candidate vertices for splitting an edge $e$ is taken from the union of the vertices in $\mathcal{M}$ associated with the two triangles incident to $e$. When this set is empty, the edge is not

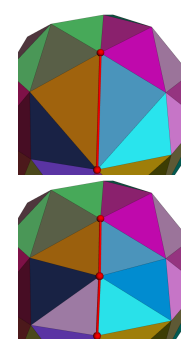

(a)

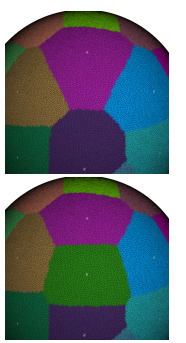

(b)

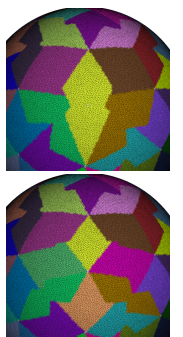

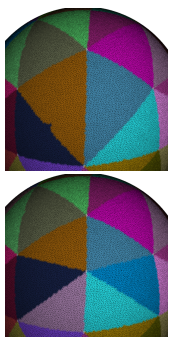

(d)
Figure 7: Mapping between the original mesh $\mathcal{M}$ and the reconstructed mesh $\mathcal{M}_{i}$. Top row : before edge insertion. Bottom row : after insertion. (a) the reconstructed mesh $\mathcal{M}_{i}$, with colored triangles. (b) geodesic Voronoi Diagram on $\mathcal{M}$, with the vertices of $\mathcal{M}_{i}$ as Voronoi Sites. (c) mapping between the vertices of $\mathcal{M}$ and the edges of $\mathcal{M}_{i}$. (b) mapping between the vertices of $\mathcal{M}$ and the triangles of $\mathcal{M}_{i}$.

split. When the set is not empty, we pick the vertex which is the closest to the optimal representative vertex, computed using Quadric Error Metrics, such as proposed by Garland and Heckbert [GH97].

We conjecture that this algorithm guarantees a good vertices selection for convex objects, and we expect its efficiency to decrease when the difference between the original mesh $\mathcal{M}$ and the base mesh increases, i.e. when the cross-parametrization between $\mathcal{M}$ and $\mathcal{M}_{b}$ exhibits severe distortions. A typical example is a protrusion or a concave region not well represented in the base mesh. More precisely, the way the encoder choses the new vertex for insertion in the reconstructed mesh $\mathcal{M}_{i}$ depends mostly on the geodesic Voronoi diagram constructed on $\mathcal{M}$. However, the adjacency of the created Voronoi regions and the connectivity of $\mathcal{M}_{i}$ sometimes differ, and this can result in a bad choice for the vertex insertion.

\subsection{Fixing connectivity drifts}

With the increase in resolution, the difference in connectivity between $\mathcal{M}$ and $\mathcal{M}_{i}$ can also increase, which can cause normal flips on the reconstructed surface, and a mesh whose connectivity differs a lot from the original mesh connectivity, thus reducing the efficiency of our approach.

To correct these potential connectivity drifts, before each edge split, we perform additional comparisons between the connectivity of $\mathcal{M}_{i}$ and the connectivity of the underlying geodesic Voronoi diagram. Let us assume that $e$ is the edge to split, with $v_{1}$ and $v_{2}$ as its vertices, $v_{3}$ and $v_{4}$ as its opposite vertices, as shown in figure 8.(c). The vertex chosen for insertion is noted $v_{5}$, and it results in the creation of a new Voronoi region $V_{5}$ in the geodesic Voronoi diagram. As a rule of thumb, we flip an edge between a parent vertex $\left(v_{1}\right.$ or $\left.v_{2}\right)$ and an opposite vertex $\left(v_{3}\right.$ or $\left.v_{4}\right)$ when the opposite 


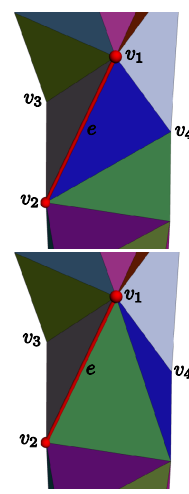

(a)

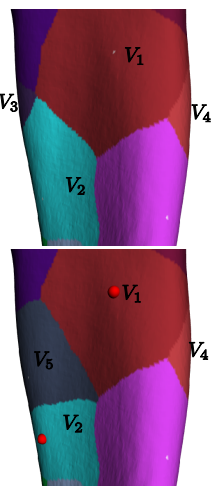

(b)

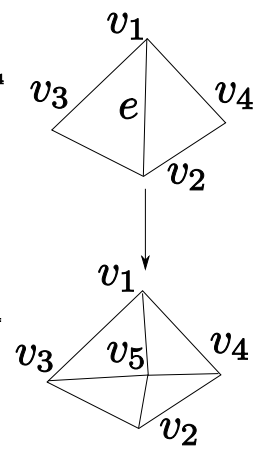

(c)

Figure 8: The reconstructed mesh connectivity $\mathcal{M}_{i}((a)$, top $)$ does not conform the connectivity of the geodesic Voronoi diagram on $\mathcal{M}\left((b)\right.$, top, one region for each vertex of $\left.\mathcal{M}_{i}\right)$. We aim at keeping this difference small. In this case, the edge e is the split location. However, the edge $v_{2} v_{4}$ is flipped before splitting the edge $e$, as the Voronoi region $V_{4}$ is neither adjacent to $V_{2}$ nor to the Voronoi region $V_{5}$ resulting from the insertion of the vertex $v_{5}$. This flip reduces the difference between the connectivity of $\mathcal{M}_{i}((a)$, bottom) and the adjacency graph of the Voronoi diagram when $v_{5}$ is inserted $((b)$, bottom). (c) the edge split mask

vertex Voronoi region $\left(V_{3}\right.$ or $\left.V_{4}\right)$ is connected to none of the Voronoi regions $V_{5}$ and the parent vertex region $\left(V_{1}\right.$ or $\left.V_{2}\right)$. An example is shown in figure 8 . This operation also has to be performed by the decoder, and therefore represents overhead data to be transmitted. As a consequence, each midpoint code can have the value 0,1 or 2 , where 2 means that additional edge flips are to be performed before the edge split. In case of a ' 2 ' value, 4 bits need to be transmitted in order to know which edges have to be flipped. In our experiments, the amount of information needed for these incident flips never exceeds $0.2 \mathrm{bpv}$. Note that the introduction of a more robust vertices picking algorithm will alleviate the need for such a workaround.

\subsection{Base mesh construction and encoding}

In order to create the base mesh $\mathcal{M}_{b}$ from the original mesh $\mathcal{M}$, we use an algorithm similar to Garland and Heckbert's approach [GH97], where the edge collapse order is determined by measuring the respective geometric degradation generated by each collapse. As we want the base mesh vertices to be a subset of the original mesh vertices, when collapsing an edge $e$ with vertices $v_{1}$ and $v_{2}$, we do not compute an optimal vertex position for the representative vertex, but choose the best position between $v_{1}$ and $v_{2}$. Moreover, we prevent edge collapses that cause normal flips on the surface or which change the topology of the mesh. For each model, the number of base mesh vertices $b$ is arbitrarily chosen, with

\begin{tabular}{|c||c||c|c|c|c||c|}
\hline \multicolumn{1}{|c||}{} & \multicolumn{1}{|c||}{} & \multicolumn{4}{c||}{ Compression } & Dec. \\
\hline Model & \#v & I & II & III & total & total \\
\hline Fandisk & 6475 & 0.1 & 0.4 & 0.3 & 0.8 & 0.2 \\
\hline Horse & 19851 & 0.7 & 1.4 & 1.7 & 3.8 & 0.5 \\
\hline Torus & 36450 & 0.8 & 3.1 & 1.2 & 5.1 & 1.1 \\
\hline Rabbit & 67039 & 2 & 7 & 5 & 14 & 2 \\
\hline Fertility & 241607 & 8 & 25 & 27 & 60 & 9 \\
\hline
\end{tabular}

Table 1: Timings for compression and decompression (in seconds), where the compression is split into three parts : Base Mesh construction (I), mesh refinement (II) and Postprocessing edge flips (III)

the objective of constructing a base mesh sufficiently close to the original mesh, with as few vertices as possible. When the vertices selection fails to choose the good order of vertices insertion, one can increase $b$, so as to reduce the difference between $\mathcal{M}_{b}$ and $\mathcal{M}$. Currently, our algorithm encodes the base mesh vertices by simply storing the vertices coordinates with a fixed resolution of 4 bits per vertex, which is automatically refined using the quantization criterion defined in section 4.3. The base mesh connectivity is also simply encoded by storing for each triangle the indexes of its three vertices. Note that a better compression efficiency will be reached using a coder such as the approach of Touma and Gotsman [TG98].

\section{Experimental results}

In this section, we compare our approach with Wavemesh [VP04], the approach of Alliez \& Desbrun [AD01], the Octree Compression approach [PK05] and the single-resolution coder from Touma \& Gotsman [TG98]. Table 1 shows timings for our approach on a workstation with a quad-core Intel $\mathrm{CPU}$ running at $2.66 \mathrm{GHz}$. For the compression, we have split the timing into three parts : program initialization and base mesh construction (I), mesh refinement (II) and post-processing edge flips (III). Note the asymmetric behavior of our approach, as decompression is performed much faster than compression. In terms of memory footprint, our implementation exhibits a peak virtual memory occupation of $282 \mathrm{MB}$ for the compression of the Fertility model and $119 \mathrm{MB}$ for its decompression.

Table 2 shows lossless compression results for different reference models. All the models were quantized to 12 bits per coordinates, except for the fandisk model (quantized to 10 bits). Note that the different approaches do not have exactly equivalent quantization strategies. This results in small differences between the vertices coordinates of the reconstructed meshes with the three different approaches. Hence, the accuracy of this comparison is not maximal, albeit good enough to exhibit differences. For our coder, the connectivity bitstream consists in the set of midpoint codes, the incident flip codes used to decrease the connectivity drift and the edge flips codes used to reconstruct the exact input connectiv- 


\begin{tabular}{|c|c|c|c|c|c|c|c|c|c|c|c|c|c|c|c|}
\hline \multirow[t]{2}{*}{ Approach } & \multicolumn{3}{|c|}{ Fandisk (6475v, 10bits/c) } & \multicolumn{3}{|c|}{ Horse $(19851 \mathrm{v}, 12 \mathrm{bits} / \mathrm{c})$} & \multicolumn{3}{|c|}{ Torus $(36450 \mathrm{v}, 12 \mathrm{bits} / \mathrm{c})$} & \multicolumn{3}{|c|}{ Rabbit $(67039 \mathrm{v}, 12 \mathrm{bits} / \mathrm{c})$} & \multicolumn{3}{|c|}{ Fertility $(241607 \mathrm{v}, 12 \mathrm{bits} / \mathrm{c}$} \\
\hline & $\mathrm{C}$ & $\mathrm{G}$ & Total & $\mathrm{C}$ & G & Total & $\mathrm{C}$ & $\mathrm{G}$ & Total & $\mathrm{C}$ & $\mathrm{G}$ & Total & $\mathrm{C}$ & $\mathrm{G}$ & Total \\
\hline OC [PK05] & 2.6 & 10.7 & 13.3 & 2.9 & 13.7 & 16.6 & 2.9 & 8.9 & 11.8 & 3.4 & 11.4 & 14.8 & 2.7 & 10 & 12.7 \\
\hline $\mathrm{AD}[\mathrm{AD} 01]$ & 5 & 12.4 & 17.4 & 4.6 & 16.2 & 20.8 & 0.4 & & & & & & & & \\
\hline TG [TG98] & 1 & 9.3 & 10.3 & 2.3 & 15.2 & 17.5 & 0.004 & 4.6 & 4.6 & 1.7 & 10.7 & 12.4 & 1.8 & 11.7 & 13.5 \\
\hline
\end{tabular}

Table 2: Comparison of lossless compression efficiency between our approach and previous works. Numbers are in bits/vertex

ity. The geometry bitstream consists in the delta-coordinates (decomposed into sign and amplitude codes) and the quantization refinement bits. The cases where our approach did not outperform other coders are: (1) with the horse model, where the base mesh has 200 vertices, which causes a relatively high coding cost of about $0.9 \mathrm{bpv}$ for the base mesh, with our naive approach. But the transmission of the remaining geometry and topology is convincing, since its coding cost reduces to $17.3 \mathrm{bpv}$. (2) with the torus model, which has very regular connectivity and is then a very good fit for connectivitydriven approaches such as Wavemesh and the coder of Alliez \& Desbrun [AD01]. (3) with the fertility model, due to the final edges flip sequence accounting for more than $50 \%$ of the connectivity bitstream. Figure 9 shows the rate-distortion curves obtained when performing progressive transmission for the Fertility model (left), the horse (middle) and the rabbit (right), where the distortion is measured in terms of raw RMS distance (forward+backward) with METRO [CRS98]. As Wavemesh only provides a limited number of resolution levels, its distortion curve is represented by steps, where each step denotes an increase in resolution. For the rabbit and Fertility models, which are relatively simple models, our approach outperforms both Wavemesh and the OC coder. For the rabbit, we also provide the rate-distortion curve when using constant quantization $\left(N_{b}=Q_{m}=12\right)$. It can be noticed that adaptive quantization is beneficial at bitrates lower than $2 \mathrm{bpv}$. The results on the horse model show that our approach still performs very well at low bitrates, but is gradually outperformed by the OC coder as the bitrate increases.

\section{Discussion}

In this paper, we have proposed a novel progressive lossless compression scheme, based on Incremental Parametric Refinement, where the algorithm reconstructs visually pleasing meshes at a low coding cost by predicting the refinement locations (i.e. the edge splits) and the required precision for each vertex. The remaining changes in the mesh connectivity are performed automatically. Experimental results show that the concept behind IPR is of great interest for efficient progressive compression of 3D models, from small models of a few thousand vertices to models with a high sampling density. Our approach still leaves room for future improvements such as more fine-grained criteria (for the refinement location and for the balance between quantization and vertices density) and efficient coding of the delta-coordinates by introducing context-dependent coding schemes. Also, studying non-inform refinement schemes seems an interesting perspective. Finally, finding the always correct and optimal vertex insertion strategy is a challenging problem, involving topology, geometry and information theory. Our approach is currently a constant-topology scheme, but using non-constant-topology refinement operators such as generalized vertex splits at specific times during reconstruction could allow us to encode meshes with complex topology starting from very simple base meshes.

\section{Acknowledgements}

We thank the anonymous reviewers for their valuable comments which helped improving the quality of the paper. We also thank Arnaud Gelas for fruitful discussions on Delaunay flips. The fertility model is courtesy of the Aim@ Shape shape repository. Our implementation is based on the Visualization ToolKit (www.vtk.org).

\section{References}

[AD01] Alliez P., DESBRUn M.: Progressive encoding for lossless transmission of $3 \mathrm{~d}$ meshes. In ACM Siggraph Conference Proceedings (2001), pp. 198-205. 2, 8, 9

[AUGA07] Alliez P., Ucelli G., Gotsman C., Attene M.: Recent advances in remeshing of surfaces. In Shape Analysis and Structuring, de Floriani L., Spagnuolo M., (Eds.). Springer, 2007. 2

[CGR07] Chaine R., Gandoin P.-M., Roudet C.: Mesh Connectivity Compression Using Convection Reconstruction. In ACM Symposium on Solid and Physical Modeling (ACM SPM) (June 2007), Siggraph A., (Ed.), pp. 41-49. 3

[COLR99] Cohen-Or D., Levin D., Remez O.: Progressive compression of arbitrary triangular meshes. In IEEE Visualization 99 (1999), pp. 67-72. 2

[CRS98] Cignoni P., Rocchini C., Scopigno R.: Metro: Measuring error on simplified surfaces. Computer Graphics Forum 17, 2 (1998), 167-174. 9

[Dee95] DEERING M.: Geometry compression. In SIGGRAPH '95: Proceedings of the 22nd annual conference on Computer graphics and interactive techniques (New York, NY, USA, 1995), ACM, pp. 13-20. 1

[DZM07] Dyer R., Zhang H., Möller T.: Delaunay mesh construction. In SGP 'O7 (July 2007), Eurographics Association, pp. 273-282. 4

[GD02] Gandoin P.-M., Devillers O.: Progressive lossless compression of arbitrary simplicial complexes. In SIGGRAPH '02: Proceedings of the 29th annual conference on Computer graphics and interactive techniques (New York, NY, USA, 2002), ACM, pp. 372-379. 2, 4 

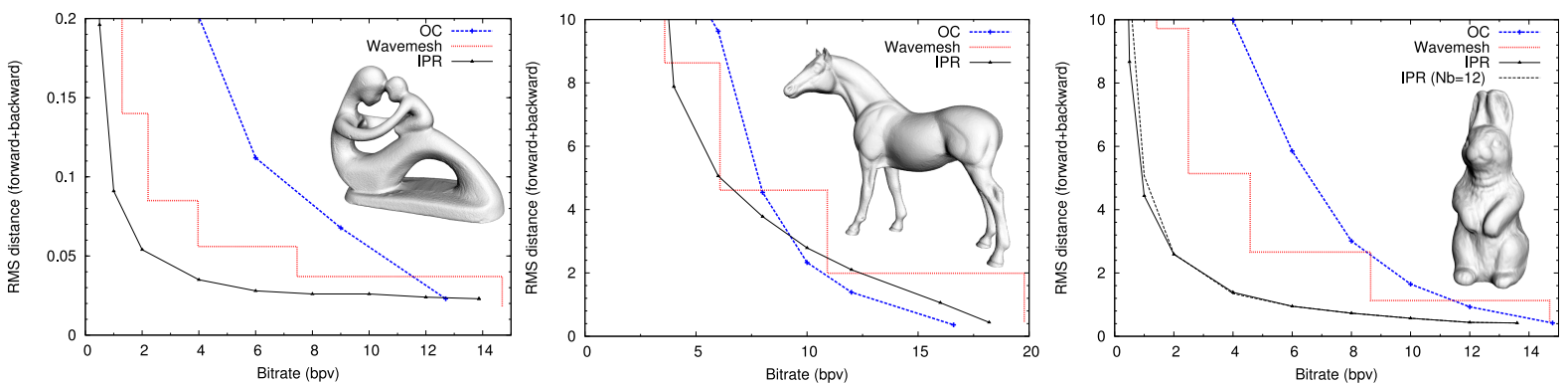

Figure 9: Comparison of rate-distortion efficiency on different models: fertility (left), horse (middle) and rabbit (right).

[GGH02] GU X., Gortler S. J., Hoppe H.: Geometry images. ACM Trans. Graph. (proceedings of SIGGRAPH 2002) 21, 3 (2002), 355-361. 2

[GH97] Garland M., Heckbert P. S.: Surface simplification using quadric error metrics. In SIGGRAPH '97: Proceedings of the 24th annual conference on Computer graphics and interactive techniques (New York, NY, USA, 1997), ACM Press/Addison-Wesley Publishing Co., pp. 209-216. 7, 8

[Got03] Gotsman C.: On the optimality of valence-based connectivity coding. Computer Graphics Forum 22 (March 2003), 99-102(4). 1

[GUW01] Gao Z., URrutia J., Wang J.: Diagonal flips in labelled planar triangulations. Graphs Combin 17 (2001), 647657. 6

[GVSS00] GUSKOV I., VIDIMČE K., SWELdENS W., SCHRÖDER P.: Normal meshes. In SIGGRAPH 'OO: Proceedings of the 27th annual conference on Computer graphics and interactive techniques (New York, NY, USA, 2000), ACM Press/Addison-Wesley Publishing Co., pp. 95-102. 2

[Hop96] Hoppe H.: Progressive meshes. In ACM Siggraph 96 Conference Proceedings (1996), pp. 99-108. 2

[ILS05] Isenburg M., Lindstrom P., Snoeyink J.: Lossless compression of predicted floating-point geometry. JCAD Journal for Computer-Aided Design 37 (2005), 2005. 2

[KBG02] Karni Z., Bogomjakov A., Gotsman C.: Efficient compression and rendering of multi-resolution meshes. In VIS '02: Proceedings of the conference on Visualization '02 (Washington, DC, USA, 2002), IEEE Computer Society, pp. 347-354. 2

[KG00] Karni Z., Gotsman C.: Spectral Compression of Mesh Geometry. In ACM Siggraph 00 Conference Proceedings (2000), pp. 279-286. 2

[Kob00] Kobbelt L.: $\sqrt{3}$-subdivision. In SIGGRAPH '00: Proceedings of the 27th annual conference on Computer graphics and interactive techniques (New York, NY, USA, 2000), ACM Press/Addison-Wesley Publishing Co., pp. 103-112. 2

[KR99] KInG D., RossignaC J.: Optimal Bit Allocation in 3D Compression. Journal of Computational Geometry, Theory and Applications 14 (1999), 91-118. 3

[KSS00] KhOdAKOVSKY A., Schröder P., SWEldens W.: Progressive Geometry Compression. ACM Siggraph Conference Proceedings (2000), 271-278. 2

[LCL*06] Lewiner T., Craizer M., Lopes H., Pesco S., VElHo L., MedeIRos E.: Gencode: geometry-driven compression for general meshes. Computer Graphics Forum 25, 4 (december 2006), 685-695. 2
[LSS*98] LEE A. W. F., SWEldens W., SChröder P., COWSAR L., DoBKIN D.: Maps: multiresolution adaptive parameterization of surfaces. In SIGGRAPH '98: Proceedings of the 25th annual conference on Computer graphics and interactive techniques (New York, NY, USA, 1998), ACM, pp. 95-104.

[PA05] PAYAN F., Antonini M.: An efficient bit allocation for compressing normal meshes with an error-driven quantization. Comput. Aided Geom. Des. 22, 5 (2005), 466-486. 2

[PK05] PENG J., KUO C.-C. J.: Geometry-guided progressive lossless $3 \mathrm{~d}$ mesh coding with octree (ot) decomposition. ACM Trans. Graph. 24, 3 (2005), 609-616. 2, 3, 4, 8, 9

[PM05] Peyré G., Mallat S.: Surface compression with geometric bandelets. In SIGGRAPH '05: ACM SIGGRAPH 2005 Papers (New York, NY, USA, 2005), ACM, pp. 601-608. 2

[PR00] Pajarola R., RossignaC J.: Compressed Progressive Meshes. IEEE Transactions on Visualization and Computer Graphics 6(1) (2000), 79-93. 2

[PS06] Poulalhon D., Schaeffer G.: Optimal coding and sampling of triangulations. Algorithmica 46, 3 (2006), 505-527.

[Ros99] RossignaC J.: EdgeBreaker : Connectivity Compression for Triangle Meshes. IEEE Transactions on Visualization and Computer Graphics (1999). 1

[SAPH04] Schreiner J., Asirvatham A., Praun E., Hoppe H.: Inter-surface mapping. ACM Transactions on Graphics (proceedings of SIGGRAPH 2004) 23 (2004), 870-877. 7

[SP96] Said A., Pearlman W.: A new, fast, and efficient image codec based on set partitioning in hierarchical trees. IEEE Transactions on Circuits and Systems for Video Technology 6, 3 (June 1996), 243-250. 3

[TG98] Touma C., Gotsman C.: Triangle Mesh Compression. Graphics Interface 98 Conference Proceedings (1998), 26-34. 1, 8,9

[TGHL98] Taubin G., GuÉziec A., Horn W., LAZARus F.: Progressive Forest Split Compression. In ACM Siggraph 98 Conference Proceedings (1998), pp. 123-132. 2

[Tut62] TutTE W.: A Census of Planar Triangulations. Canadian Journal of Mathematics 14 (1962), 21-38. 1

[VP04] VAlette S., Prost R.: A wavelet-based progressive compression scheme for triangle meshes : Wavemesh. IEEE Trans Visu Comp Grap 10, 2 (2004), 123-129. 2, 8, 9

[Wag36] WAGNER K.: Bemerkung zum vierfarbenproblem. Jber. Deutsch. Math.-Verein. 46 (1936), 26âĂŞ32. 6 